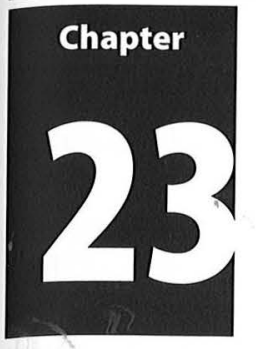

\title{
Sperm selection for assisted conception
}

\author{
R. J. Aitken
}

\section{Introduction}

The development of safe, effective, efficient methods for the isolation of human spermatozoa is a major feature of current research into the optimization of assisted conception therapy. The objective of such research is to deliver methods that will efficiently generate populations of motile, functional spermatozoa that are competent to fertilize the oocyte in vitro and initiate normal embryo development. If sperm quality is so seriously impaired that conventional fertilization is impossible, then the sperm isolation procedure should be competent to recover spermatozoa that are, at least, capable of supporting normal embryonic development following intracytoplasmic sperm injection (ICSI).

The methods that have been used to isolate sperm populations for assisted conception purposes include: (1) repeated cycles of centrifugation (typically $600 \mathrm{~g}$ for five minutes) and resuspension with a simple culture medium; (2) swim-up from a washed pellet; (3) swim-up (or swim down) from semen followed by a centrifugation and resuspension step to remove any traces of seminal plasma; (4) centrifugation through discontinuous density gradients composed of media such as Percoll ${ }^{\circ}$ (colloidal silica coated with polyvinyl pyrrolidone), Puresperm ${ }^{\circledR}$ or ISolate ${ }^{\circledR}$ (colloidal silica with covalently bound silane), and (5) a recently introduced electrophoretic method $[1,2]$. In addition, a variety of procedures, including filtration through columns containing glass wool, glass beads, or Sephadex, are available for further purifying the sperm suspensions once they have been isolated using the above techniques. In addition, treatment with magnetic Dynabeads ${ }^{\circledR}$ or ferrofluids coated with anti-CD45 monoclonal antibodies has been used to rid sperm suspensions of contaminating leukocytes [3]. Assessment of which of these techniques is the most appropriate for clinical application depends upon an understanding of the fundamental principles underpinning their effectiveness as sperm isolation strategies.

\section{Basic principles of sperm isolation}

Fundamental to the process of sperm isolation is an awareness of the vulnerability of human spermatozoa to oxidative stress. These cells are vulnerable to such damage because they are well endowed with targets for oxidative attack, particularly polyunsaturated fatty acids (50\% of the fatty acid in a human sperm cell is docosahexaenoic acid with six double bonds per molecule) and DNA. The vulnerability of these highly specialized cells is further compounded by the limited volume and restricted distribution of cytoplasmic space which, in 
somatic cells, would house critical antioxidant defence enzymes such as catalase, superoxide dismutase, or glutathione peroxidase $[1,4]$. Because of this inherent vulnerability, spermatozoa are highly reliant on the antioxidant properties of the extracellular fluids in which they are bathed to provide antioxidant protection. It is for this reason that seminal plasma has evolved into one of the most powerful antioxidant fluids known to man.

Such antioxidant protection is necessary because, at the moment of ejaculation, spermatozoa come into contact with leukocytes for the very first time. Although the source of these leukocytes is still the subject of debate, it is highly likely that they enter the seminal plasma via the urethra and secondary sexual glands. Every human semen sample is contaminated with leukocytes, a majority of which are neutrophils and macrophages that are actively generating reactive oxygen species (ROS) [5]. Thus, whatever sperm isolation protocols we use we should try to ensure that spermatozoa and leukocytes do not come into contact with each other without the protection afforded by seminal plasma. It is for this reason that sperm preparation techniques that involve the isolation of spermatozoa directly from seminal plasma, whether this is discontinuous gradient centrifugation, swin-up from unfractionated semen, or electrophoretic separation, usually generate sperm populations that are more motile and functional than those prepared by repeated centrifugation or swim-up from a washed pellet $[1,6]$. With the latter techniques, the spermatozoa are suddenly exposed to a free radical attack originating from the seminal leukocyte population in the absence of antioxidant protection. Furthermore, some culture media, such as Hams F10, are supplemented with transition metals, such as iron, that only exacerbate the oxidative stress experienced by the spermatozoa by promoting the lipid peroxidation reactions that have such a devastating effect on sperm function [7].

Even when sperm populations have been isolated direct from seminal plasma on Percoll gradients, or to a lesser extent by swim-up, they may still be contaminated with leukocytes. A detailed assessment of leukocyte contamination in sperm preparations submitted for ART demonstrated that around $30 \%$ of such preparations were still contaminated with leukocytes. Moreover the level of leukocyte contamination detected in such samples was negatively associated with fertilization rates following IVF [8].

If, even after preparing sperm samples directly from semen in the recommended manner, leukocyte contamination is still a problem, there are three major courses of action that might be pursued:

1) detect the leukocytes using a simple, sensitive chemiluminescent assay employing formyl peptides or opsonized zymosan as a stimulant and then, if these cellular contaminants are present, use magnetic beads coated in anti-CD45 (the common leukocyte antigen) to effect their removal [3].

2) Incorporate antioxidants into the medium to counteract the negative impact of leukocyte contamination. In this context, glutathione, $\mathrm{N}$-acetylcysteine, hypotaurine, and catalase have all been shown to exert a protective effect in vitro [9].

3) Supplement the medium with reagents such as EDTA, DETAPAC or apotransferrin in order to chelate cytotoxic transition metals and neutralize the stimulatory impact of ROS on lipid peroxidation.

\section{DNA damage}

In times past, it was envisaged that the only attribute of sperm quality that had to be preserved during sperm isolation was the capacity of these cells for fertilization. We now know that another important property that spermatozoa must confer upon the zygote is the capacity for normal embryonic development. In this context a variety of factors may be important including: (1) a functional centrosome to regulate cell division in the embryo, (2) remodelled chromatin characterized by the presence of protamines 1 and 2 in a ratio of 1:1, as well as appropriately modified histones, (3) a population of mRNA and miRNA species that are carried into the zygote by the fertilizing spermatozoon and may contribute to the regulation of early embryonic development, (4) an appropriate pattern of DNA methylation, and (5) intact nuclear DNA.

The latter is thought to be a particularly important aspect of sperm quality. DNA damage in spermatozoa has been linked with a wide variety of adverse clinical outcomes including impaired fertilization, disrupted pre-implantation development, an increased risk of miscarriage, and a wide variety of defects in the offspring ranging from complex neurological conditions such as autism or spontaneous schizophrenia to childhood cancer [4].

Although definitive evidence is lacking, it is possible that DNA damage in the male germ line makes a significant contribution to the elevated incidence of birth defects observed following ART, when compared with the naturally conceived population. Furthermore, there is good evidence for an increase in imprinting disorders, notably the Beckwith-Wiedemann and Angelman syndromes, in children produced by assisted conception, which may be associated with defects in the methylation status of spermatozoa. It is also known that singleton infants produced by ART are significantly more likely to be admitted to a neonatal intensive care unit, to be hospitalized, and to stay in hospital tonger than their naturally conceived counterparts. In addition, recent independent studies have revealed an eight-fold increase in the incidence of undescended testicles in boys conceived by ICSI, while another analysis demonstrated abnormal retinal vascularization in such children [4]. In order to negate the male contribution to such abnormalities, the spermatozoa we isolate for assisted conception purposes should not only be capable of fertilization but also possess as little DNA damage as possible.

In considering the impact of sperm preparation techniques on sperm DNA integrity, it is important to stress that a majority of the DNA damage we see in the spermatozoa of male patients is, like defective sperm function, oxidatively induced. DNA damage is also tightly correlated with defects in the remodelling of sperm chromatin during spermiogenesis [10]. On the basis of these results, we have proposed a two-step hypothesis for the induction of DNA damage in the male germ line [4]. In the first step, spermiogenesis becomes disrupted leading to the generation of spermatozoa possessing poorly remodelled chromatin and lacking a full complement of protamines. In the second step, these vulnerable cells become oxidatively attacked leading to the rapid formation of base adducts, particularly 8-hydroxy$2^{\prime}$-deoxyguanosine. The formation of such adducts then destabilizes the DNA backbone leading to the formation of abasic sites and, ultimately, the appearance of DNA strand breaks.

Thus whatever approach we adopt in the preparation of spermatozoa for ART, it should avoid the formation of oxidative DNA damage. A number of studies have demonstrated that the preparation of spermatozoa by, for example, discontinuous gradient centrifugation produces populations possessing low levels of DNA damage [11]. Although this is a positive finding, it should be noted that such assessments of sperm DNA integrity with TUNEL or Comet assays do not take account of cell viability. A recent technical development in this area has, for the first time, permitted the simultaneous assessment of DNA damage and cell vitality by flow cytometry [12]. Application of this methodology clearly demonstrated that a majority of the DNA damaged spermatozoa found in the ejaculate are, in fact, dead. This study also confirmed previous reports indicating that discontinuous gradient centrifugation 
leads to the production of sperm suspensions exhibiting significantly more vitality and (as a result) significantly less DNA damage than unfractionated sperm suspensions. However when only the viable cells were considered, discontinuous gradient centrifugation was shown to actually induce a significant increase in oxidative DNA damage that, in the patient population, translated into an increase in the levels of DNA fragmentation [12]. Thus, even when care is taken to isolate spermatozoa using state-of-the-art preparation techniques, DNA damage may still be induced in these cells. New technologies are clearly needed.

\section{Electrophoretic sperm isolation}

Ainsworth et al. described an alternative method of isolating spermatozoa for assisted conception purposes in 2005 [6]. This procedure relied upon two fundamental principles: (1) that normal functional spermatozoa are negatively charged and thus move towards the anode in an electric field and (2) that spermatozoa are amongst the smallest cells in the body and will pass through a $5 \mu \mathrm{m}$ filter. The electrophoretic device described in the above publication uses electric current to pull negatively charged spermatozoa through a polycarbonate filter and into a collection chamber. The separation system is depicted in Figure 23.1 and consists of a cassette comprising two $400 \mu \mathrm{l}$ chambers separated by a polycarbonate filter containing $5 \mu \mathrm{m}$ pores and bounded by a $15 \mathrm{kDa}$ polyacrylamide membrane to allow the free circulation of buffer. Semen is introduced into one chamber, current applied (75 mA at variable voltage) and within seconds a purified suspension of spermatozoa can be collected from the adjacent chamber in an appropriate buffer.

The method is extremely rapid, and isolates sperm populations possessing good motility, excellent morphology, and low rates of DNA damage. Furthermore these cell populations are essentially free of contamination by leukocytes and precursor germ cells. The method is also effective with a wide range of starting materials including testicular biopsies and cryostorage media [6].

Given the initial promise of this system, it was employed in a case study of a couple experiencing prolonged infertility associated with high rates of DNA damage in the male partner's spermatozoa [13]. In this example, the patient produced an oligozoospermic ejaculate on the day of oocyte retrieval containing 3.2 million spermatozoa/ml and an equivalent number $(2.1 \mathrm{million} / \mathrm{ml})$ of contaminating round cells. Of the spermatozoa that were present, $30 \%$ were vital, $18 \%$ were motile and $26 \%$ were TUNEL positive. Following electrophoretic separation, a purified sperm population was generated that was $62 \%$ vital, $24 \%$ motile and $14 \%$ TUNEL positive. Assessment of these samples by SCSA revealed a DNA fragmentation index that fell from $41 \%$ in the ejaculated sample to $15 \%$ in the electrophoretically separated population. The isolated spermatozoa were then used in ICSI; five out of seven oocytes were fertilized, and two of the embryos were transferred on day 3. Embryo transfer was followed by pregnancy and, later, the birth of a healthy girl with no complications.

This case report was then followed up by a more extensive clinical trial in order to compare the electrophoretic method with conventional density gradient centrifugation in a clinical setting [14]. Each semen sample was randomly split between preparation using the electrophoretic system and preparation by standard density gradient centrifugation. Furthermore each cohort of oocytes was split for insemination using either electrophoretically- or densitygradient prepared spermatozoa. In the event, both methods of sperm preparation yielded comparable rates of sperm recovery, motility, and DNA fragmentation. Furthermore there



(b)

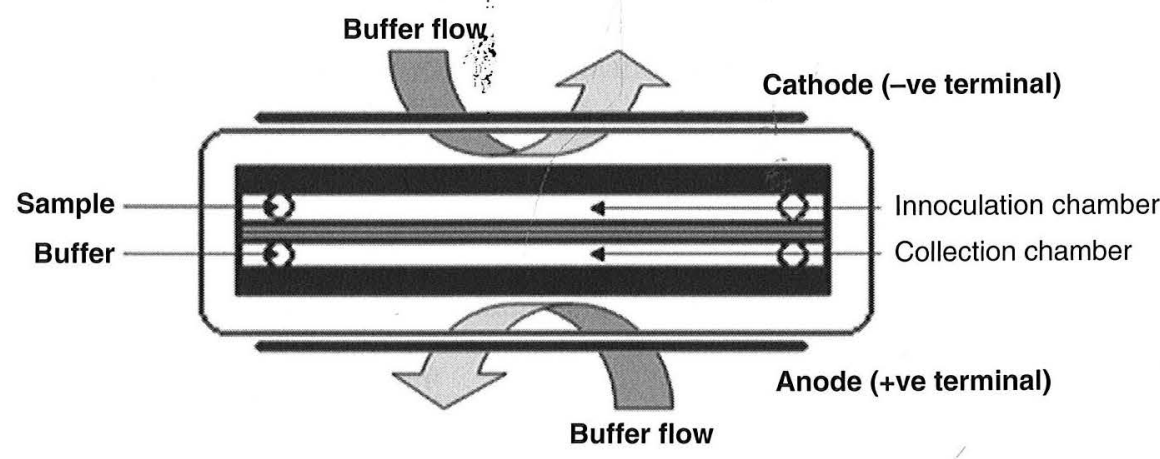

Figure 23.1 Schematic representation of the cartridge-based electrophoretic separation technology. (a) Directiona movement of competent spermatozoa in the applied electric field and resultant passage through polycarbonate $5 \mu \mathrm{m}$ separation membranes results in the rapid production of highly purified sperm populations. (b) Topography of the cartridge configuration inclualing restriction and separation menbranes, indicating buffer llows and sample

were no significant differences between these methods in the ability of the spermatozoa to achieve fertilization $(62.4 \%$ versus $63.6 \%$ ), cleavage $(99.0 \%$ versus $88.5 \%$ ), and high-quality embryos (27.4\% versus $26.1 \%$ ). Thus, this novel approach to sperm isolation appears to be as effective a sperm preparation regime as density gradient centrifugation, while taking a fraction of the time.

\section{Conclusion}

In order to ensure high rates of fertilization and normal embryonic development to term, it is essential that oocytes are inseminated with carefully prepared sperm suspensions. The latter should be free of cellular contamination and comprise only the most viable, motile, functional spermatozoa in the ejaculate. It is also extremely important that the selection technique isolates cells that are, as far as possible, free of DNA damage. In order to avoid the iatrogenic 
induction of damage to spermatozoa during their preparation, it is also important to isolate these cells directly from seminal plasma. This can be easily achieved by allowing the cells to swim out of seminal plasma, providing the intrinsic motility of the spermatozoa is adequate to the task. Alternatively, the spermatozoa can be isolated from semen using discontinuous gradient centrifugation or a new electrophoretic approach. Preliminary results suggest that the latter represents an extremely rapid, effective means of isolating high quality cells for insemination. Additional clinical trials are now needed to substantiate this early promise.

\section{Acknowledgments}

Grateful thanks are due to the ARC, Newcastle Innovation, the ARC Centre of Excellence in Biotechnology and Development and NuSep for financial support.

\section{References}

1. Aitken RJ, Clarkson JS. Significance of reactive oxygen species and antioxidants in defining the efficacy of sperm preparation techniques. J Androl 1988;9:367-76.

2. Mortimer D. Sperm preparation methods. J Androl 2000;21:357-66.

3. Aitken RJ, Buckingham DW, West K, Brindle J. On the use of paramagnetic beads and ferrofluids to assess and eliminate the leukocytic contribution to oxygen radical generation by human sperm suspensions. Am J Reprod Immunol 1996;35:541-51.

4. Aitken RJ, De Iuliis GN, McLachlan RI. Biological and clinical significance of DNA damage in the male germ line. Int J Androl 2009;32:46-56.

5. Aitken RJ, Baker HW. Seminal leukocytes: passengers, terrorists or good samaritans? Hum Reprod 1995;10:1736-9.

6. Ainsworth C, Nixon B, Aitken RJ. Development of a novel electrophoretic system for the isolation of human spermatozoa. Hum Reprod 2005;20:2261-70.

7. Gomez E, Aitken J. Impact of in vitro fertilization culture media on peroxidative damage to human spermatozoa. Fertil Steril 1996;65:880-2.

8. Krausz C, Mills C, Rogers S, Tan SL, Aitken RJ. Stimulation of oxidant generation by human sperm suspensions using phorbol esters and formyl peptides: relationships with motility and fertilization in vitro. Fertil Steril 1994;62:

599-605.
9. Baker HW, Brindle J, Irvine DS, Aitken RJ. Protective effect of antioxidants on the impairment of sperm motility by activated polymorphonuclear leukocytes. Fertil Steril 1996;65:411-9.

10. De Iuliis GN, Thomson LK, Mitchell LA, et al. DNA damage in human spermatozoa is highly correlated with the efficiency of chromatin remodeling and the formation of 8-hydroxy-2'-deoxyguanosine, a marker of oxidative stress. Biol Reprod 2009;81:517-24.

11. Donnelly ET, O'Connell M, McClure N, Lewis SE. Differences in nuclear DNA fragmentation and mitochondrial integrity of semen and prepared human spermatozoa. Hum Reprod 2000;15:1552-61.

12. Mitchell LA, De Iuliis GN, Aitken RJ. The TUNEL assay consistently underestimates DNA damage in human spermatozoa and is influenced by DNA compaction and cell vitality: development of an improved methodology. Int J Androl 2011;34:2-13.

13. Ainsworth C, Nixon B, Jansen RP, Aitken RJ. First recorded pregnancy and normal birth after ICSI using electrophoretically isolated spermatozoa. Hum Reprod 2007;22:197-20.

14. Fleming SD, Ilad RS, Griffin AM, et al. Prospective controlled trial of an electrophoretic method of sperm preparation for assisted reproduction: comparison with density gradient centrifugation. Hum Reprod 2008;23:2646-51. 